\title{
Identification of lymphatic pathway involved in the spread of bladder cancer: Evidence obtained from fluorescence navigation with intraoperatively injected indocyanine green
}

\author{
Shogo Inoue, MD, ${ }^{*}$ Hiroaki Shiina, MD; ${ }^{*}$ Yozo Mitsui, MD; ${ }^{*}$ Hiroaki Yasumoto, MD; ${ }^{*}$ Akio Matsubara, MD; \\ Mikio Igawa, $M D^{*}$
}

*Department of Urology, Shimane University School of Medicine, Izumo, Japan; †Department of Urology, Graduate School of Biomedical Sciences, Hiroshima University, Hiroshima, Japan

Cite as: Can Urol Assoc J 2013;7(5-6):e322-8. http://dx.doi.org/10.5489/cuaj.12096

Published online May 13, 2013 (early released September 10, 2012).

\section{Abstract}

Introduction: We identify lymphatic vessels draining from the bladder by using fluorescence navigation (FN) system.

Methods: In total, 12 candidates for radical cystectomy and pelvic lymph node dissection (PLND) were included in this study. After an indocyanine green (ICG) solution was injected into the bladder during radical cystectomy, lymphatic vessels draining from the bladder were analyzed using a FN system. PLND was based on the lymphatic mapping created from the FN measurements (in vivo probing) in the external iliac, obturator and internal iliac regions; after PLND, the fluorescence of the removed lymph nodes (LNs) was analyzed on the bench (ex vivo probing).

Results: There were no patients with complications associated with the intravesical ICG injection. A lymphatic pathway along inferior vesical vessels to internal iliac LNs was clearly illustrated in 7 cases. Under in-vivo probing, the fluorescence intensity of internal iliac nodes was greater than that of external iliac or obturator nodes. Under ex-vivo probing, the fluorescence intensity of internal iliac and obturator nodes was greater than that of external iliac nodes. Conclusions: Using an FN system after injecting ICG during a radical cystectomy operation is a safe and rational approach to detecting the lymphatic channel draining from the bladder.

\section{Introduction}

The sentinel lymph node (SLN) is the first lymph node (LN) through which cancer cells are detected from the primary site. The SLN concept is based on the hypothesis that secondary cancer cells are not found in LNs downstream from the first one receiving drainage from the primary tumour. The validity of this concept is well-established in breast and skin cancers, ${ }^{1,2}$ and it has also been applied to penile cancer by Cabanas. ${ }^{3}$ Liedberg and colleagues reported the possibility of applying the SLN concept to bladder cancer using $\gamma$ probe-guided SLN dissection, ${ }^{4}$ but their approach combining preoperative lymphoscintigraphy with an intraoperative $\gamma$ probe is complicated. Until now, two approaches have been used to identify SLNs: the dye-guided approach and the radioisotope-guided (RI-guided) approach. The dyeguided approach appears to be convenient and safe, but it sometimes cannot precisely detect SLNs deep in the pelvis, because the contrast of the target tissue is insufficient. The radioisotope-guided (RI-guided) approach can accurately determine the location of deep SLNs, but cannot identify minute lymphatic vessels. ${ }^{5}$

To identify the SLN and confirm the applicability of the SLN concept to bladder cancer, we need an anatomical understanding of the lymphatic network and flow around the bladder. The primary contributions to the knowledge of bladder lymphatics have come from European sources and were well-summarized by Leadbetter and Cooper. ${ }^{6}$ Normal vesical lymphatics drain into the perivesical network, from which originate 6 groups of pathways: (1) the visceral lymphatic plexus within the bladder wall; (2) the intercalated LNs; (3) pelvic collecting trunks; (4) regional pelvis LNs (which include the external iliac, hypogastric and sacral LNs groups); (5) lymphatic trunks originating from the regional pelvic LNs; and (6) common iliac LNs.

We have developed a novel, third method to identify SLN by using indocyanine green (ICG) fluorescence during radical cystectomy. SLN biopsy under the guidance of ICG fluorescence is considered safe and useful in diagnosing breast cancer. ${ }^{7}$ We previously found that use of a fluorescence navigation (FN) system with ICG injection was safe and convenient in determining the border between normal and malignant kidney tissues during partial nephrectomy ${ }^{8}$ and in identifying lymphatic vessels draining from the prostate; these findings validated the concept of SLNs in prostate cancer. ${ }^{9}$ However, to the best of our knowledge, there are no reports on the use of ICG fluorescence navigation for SLN mapping in bladder cancer patients. The purpose of this 
Lymphatic channel identified by fluorescence navigation

study is to demonstrate a safe and convenient approach in identifying lymphatic vessels draining from the bladder and to validate the SLN concept in bladder cancer.

\section{Methods}

\section{Patients}

We included 12 patients who were candidates for radical cystectomy and PLND. The surgery for radical cystectomy with PLND was performed between July 2007 and February 2009 at Shimane University Hospital, Japan. Written informed consent was obtained from all patients and their families after they were given a sufficient explanation according to the documentation approved by our institutional ethics committee. The median age at diagnosis was 72 years (range: 62-77 years) (Table 1).

\section{ICG injection}

A $0.5-\mathrm{mL}$ solution containing $0.25 \%$ ICG (Diagnogreen $0.25 \%$; Daiichi Pharmaceutical, Tokyo, Japan) was injected into the bladder near the tumour during radical cystectomy; within 5 minutes, lymphatic vessels draining from the bladder were visualized by the FN system. The ICG absorbs light in the near-infrared range and its peak absorption is at a wavelength of $800 \mathrm{~nm}$. It emits with maximal fluorescence at a wavelength of $840 \mathrm{~nm}$ when bound to plasma proteins. A fluorescence wavelength longer than the excitation one is specific to ICG. Autofluorescence of the pelvic tissue and blood is relatively low (in the $800-\mathrm{nm}$ wavelength range), making the signal-to-noise ratio high in ICG fluorescence imaging with the Photo Dynamic Eye (PDE) infrared camera system (Hamamatsu Photonics, Hamamatsu, Japan). The light source of this system contains 36 light-emitting diodes producing light with a wavelength of $760 \mathrm{~nm}$, and the detector is a charge-coupled-device camera equipped with a filter eliminating light with wavelengths below $820 \mathrm{~nm}$. The excitation light penetrates deeply into the pelvic tissues, and the strong fluorescence of ICG allows the detection of even small particles of ICG bound to plasma proteins in the lymphatic vessels and LNs. The fluorescence signals are transmitted to a digital video processor and displayed on a TV monitor in real time.

\section{PLND}

The LNs in three regions (external iliac, obturator fossa and internal iliac region) together with the surrounding adipose tissue were carefully dissected. If a fluorescent spot kept shining after the initial LN dissection, residual LNs were also dissected. On the basis of lymphatic mapping under FN

\begin{tabular}{lcccccc}
\hline \multicolumn{7}{l}{ Table 1. Patient characteristics } \\
\hline Case & Age & Sex & $\begin{array}{c}\text { Clinical } \\
\text { T stage }\end{array}$ & Grade & SLN & LN (mets) \\
\hline 1 & 63 & Male & T4 & 3 & + & 7 \\
2 & 65 & Male & Tis & $2>3$ & + & 6 \\
3 & 74 & Male & T1 & $2>3$ & + & 10 \\
4 & 77 & Male & T4 & 3 & - & $20(3)$ \\
5 & 66 & Male & Tis & 3 & - & $27(6)$ \\
6 & 68 & Male & T3 & $3>2$ & + & 13 \\
7 & 71 & Male & T1 & $2>3$ & + & 13 \\
8 & 77 & Male & T3 & 3 & - & $21(12)$ \\
9 & 73 & Male & T3 & $3>2$ & - & $20(17)$ \\
10 & 73 & Female & T3 & 3 & + & 14 \\
11 & 73 & Male & T3 & 3 & - & $19(5)$ \\
12 & 62 & Male & T1 & $2>3$ & + & 19 \\
\hline \multicolumn{7}{l}{ Tis: carcinoma in situ; SLN: sentinel lymph node; LN: lymph node. } \\
\hline \multicolumn{7}{c}{}
\end{tabular}

guidance (in vivo probing), PLND was performed, focusing on the external iliac, obturator and internal iliac regions. The fluorescence of the removed LNs was subsequently analyzed on the bench (ex vivo probing). The LN involvement was confirmed by hematoxylin and eosin staining of formalinfixed samples, and all LNs were pathologically examined. The fluorescence intensity of the LNs detected by the FN system was measured within a range of 0 to 255 and calculated as a relative ratio by using ImageJ (National Institutes of Health, Bethesda, MD) software on static images made from the real-time images.

\section{Statistical analysis}

All values were expressed as means, and the results were analyzed using ANOVA followed by a post-hoc test or Mann Whitney u-test. A $p$ value of less than 0.05 was considered statistically significant. The calculations were performed with the help of StatView 5.0 for the Macintosh.

\section{Results}

There were no patients with complications or adverse events associated with the intravesical ICG injection. Lymphatic pathways along inferior vesical vessels to internal iliac LNs were clearly illustrated in 7 cases, whereas other channels, such as pathways to external iliac LNs, were not prominent.

In case \#6, pathological examination did not show any $\mathrm{LN}$ metastases, and the in vivo probing with the FN system showed highly fluorescent lymphatic flow in the internal iliac region after direct injection of ICG. The lymphatic stream having ICG particles was identified as a shining stream or spot in the fluorescence image (Fig. 1, parts A, B). Rapid venous flow with immediate loss of fluorescence could be distinguished from the lymphatic stream. The highly fluorescent region was not evident after $\mathrm{LN}$ dissection. The FN 
Inoue et al.

system did not show residual LNs, and their absence was confirmed by the naked-eye examination (Fig. 1, parts C, D). LNs stained with ICG were not evident to the naked eye. In ex vivo probing with the FN system, the fluorescence of the internal iliac LN was greater than that of the external iliac or obturator LN (Fig. 1, parts E, F). Fluorescence of the LN was clearly detected for at least 2 hours.
In case \#4, though pathological examination showed LN metastases in the right external iliac and obturator LNs, there was no strong fluorescence in the regional LNs of the bladder under either in vivo or ex vivo probing (Fig. 2, parts A, B, C). Pathological examination of the bladder revealed urothelial carcinoma (grade 3), diffusely invaded lymphatic vessels (Fig. 2, parts D, E). Pathological examination also

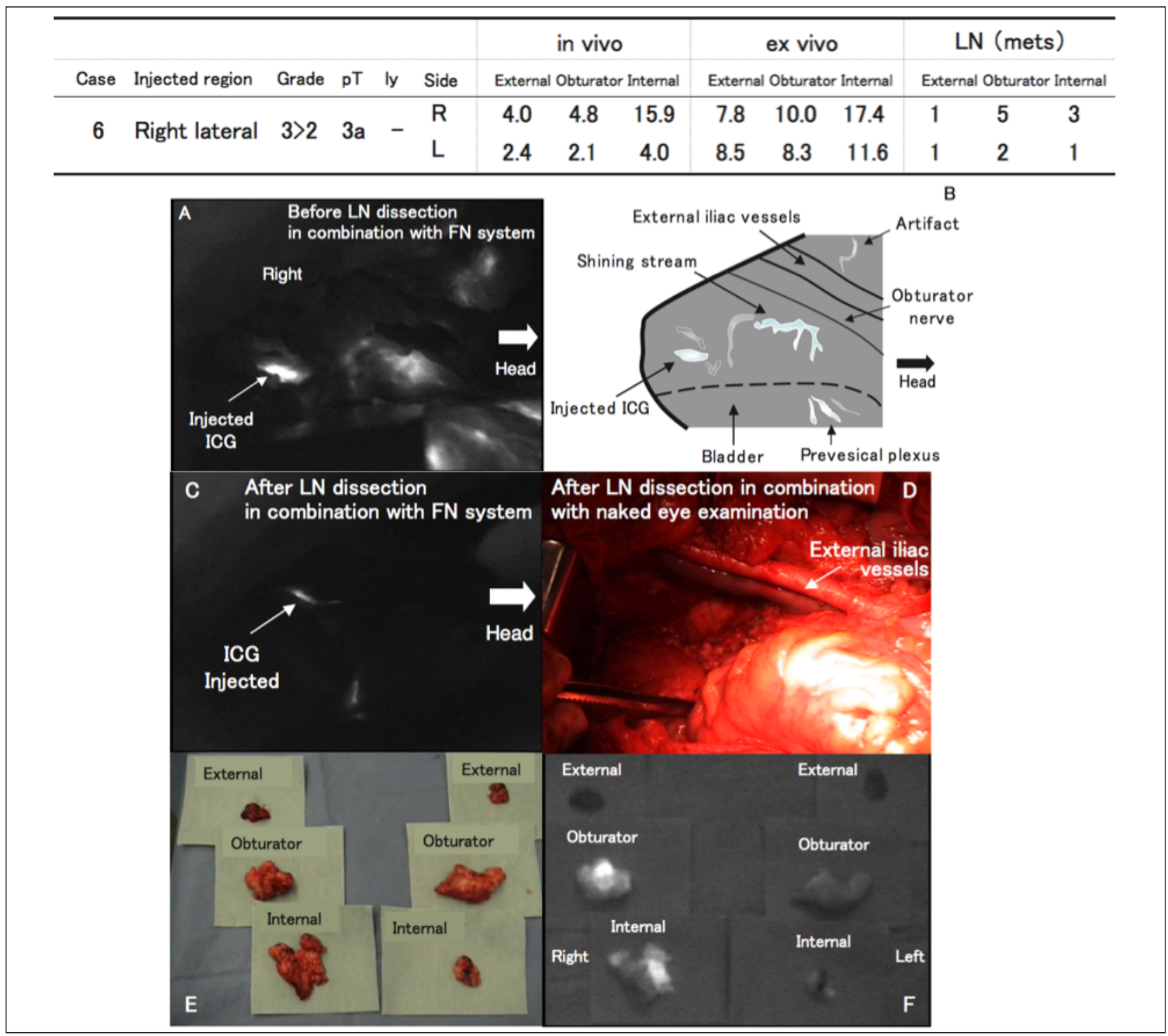

Fig. 1. Lymph node (LN) mapping in a bladder cancer patient without LN metastases identified by the fluorescence navigation system (Case \#6, right side); $A$ : Lymphatic vessels draining from the bladder and visualized by the fluorescence navigation (FN) system. The in vivo probing with the FN system showed a highly fluorescent lymphatic flow in the internal iliac region after direct injection of indocyanine green (ICG); B: Pattern diagram of lymphatic vessels visualized by the FN system; C: The highly fluorescent region was not evident in a fluorescence image taken after LN dissection; D: Naked-eye examination confirmed that there were no residual LNs; E: LNs stained with ICG were not evident to the naked eye; F: In ex vivo probing with the FN system, the fluorescence of the internal iliac and obturator LN was greater than that of the external iliac LN. 


\begin{tabular}{|c|c|c|c|c|c|c|c|c|c|c|c|c|c|c|}
\hline \multirow{4}{*}{$\begin{array}{c}\text { Case } \\
4\end{array}$} & \multirow{4}{*}{$\begin{array}{c}\text { Injected region } \\
\text { Anterior }\end{array}$} & \multirow{4}{*}{$\begin{array}{c}\text { Grade } \\
3\end{array}$} & \multirow{4}{*}{$\begin{array}{l}\text { pT } \\
4\end{array}$} & & \multirow{3}{*}{$\begin{array}{c}\text { Side } \\
\mathrm{R}\end{array}$} & \multirow{2}{*}{\multicolumn{3}{|c|}{$\begin{array}{c}\text { in vivo } \\
\text { External Obturator Internal }\end{array}$}} & \multirow{2}{*}{\multicolumn{3}{|c|}{$\begin{array}{c}\text { ex vivo } \\
\text { External Obturator Internal }\end{array}$}} & \multirow{2}{*}{\multicolumn{3}{|c|}{$\begin{array}{l}\text { LN (mets) } \\
\text { External Obturator Internal }\end{array}$}} \\
\hline & & & & ly & & & & & & & & & & \\
\hline & & & & L & & 2.2 & 2.4 & 4.8 & 5.8 & 7.3 & 4.0 & 1(1) & 4 & 6 \\
\hline & & & & + & $\mathrm{L}$ & 2.2 & 2.9 & 3.1 & 4.7 & 6.1 & 4.8 & $6(1)$ & 2(1) & 1 \\
\hline
\end{tabular}

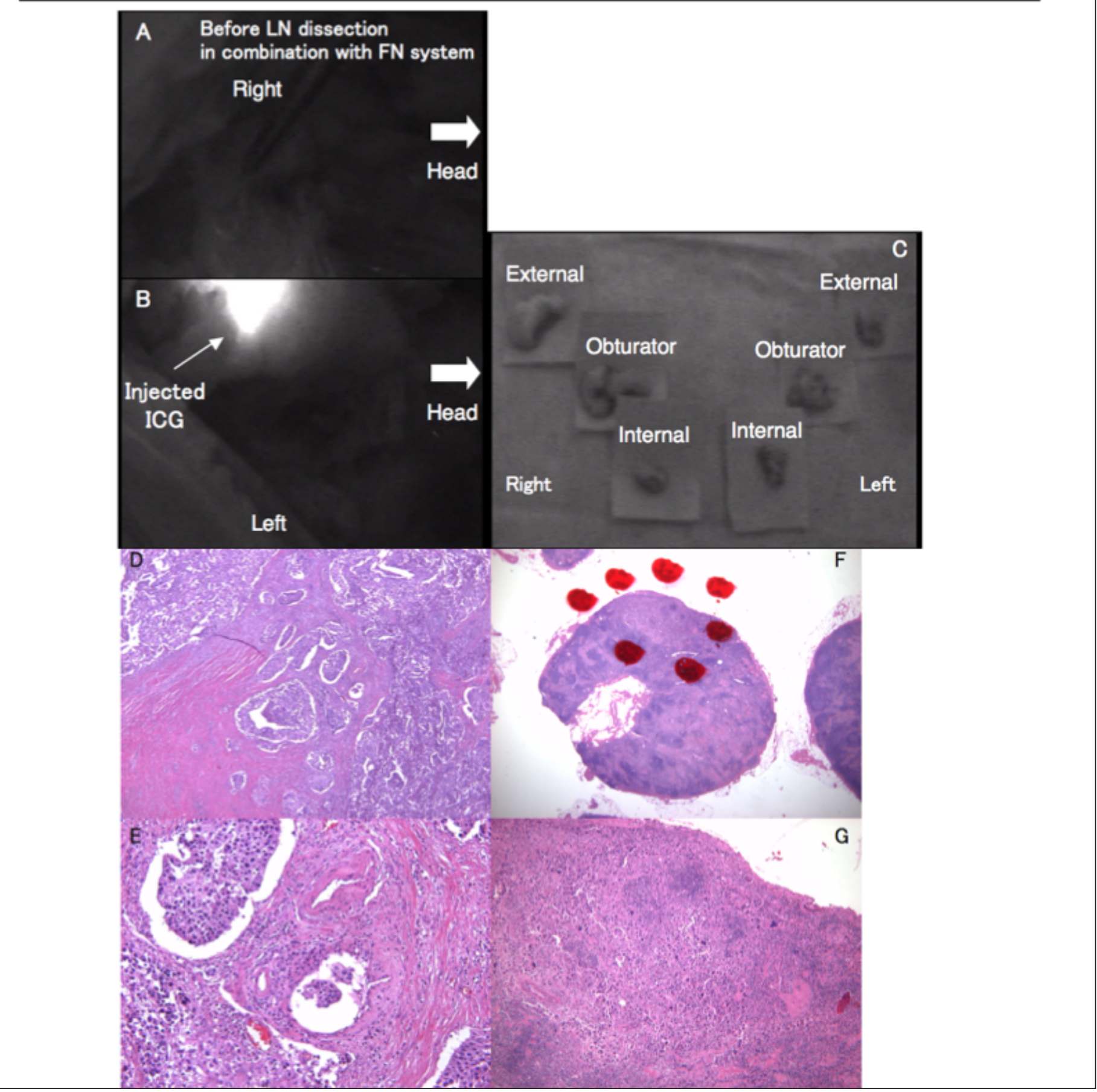

Fig. 2. Pathological lymph node (LN) involvement in relation to the lymphatic pathway identified by the fluorescence navigation (FN) system (Case \#4); $A$, B, C: Although pathological examination showed metastasis in the bilateral external iliac and left obturator LNs, there was no strong fluorescence in the regional LNs of the bladder either in vivo or ex vivo; D, E: Pathological examination revealed that urothelial carcinoma (grade 3 ) had diffusely invaded lymphatic vessels in the bladder; F, G: Urothelial carcinoma infiltrated the bilateral external and left obturator LNs. 


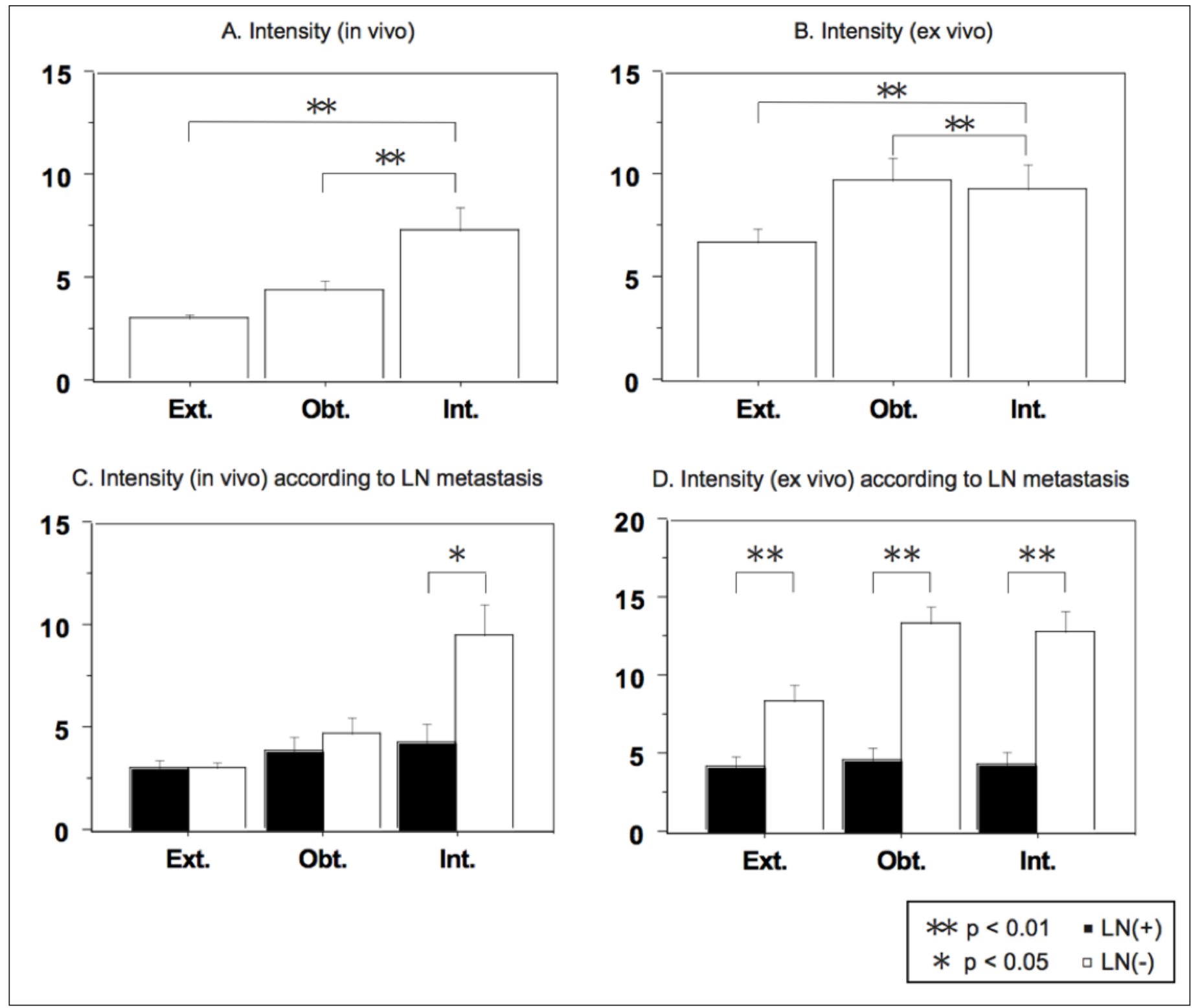

Fig. 3. Intensity of indocyanine green fluorescence in lymph nodes (LNs) determined by the luorescence navigation system; A: In the in vivo probing, the intensity difference between external and internal iliac nodes and the intensity difference between obturator and internal iliac nodes were statistically significant $(p<0.01)$; B: In the ex vivo probing, the intensities of the internal iliac and obturator nodes were significantly $(p<0.01)$ higher than that of the external iliac LNs $(p<0.01)$; C: In the in vivo probing, the intensity difference between LN metastases was statistically significant in internal iliac nodes $(p<0.05)$ but not in obturator or external iliac nodes; D: In the ex vivo probing, the intensity difference between LN metastases was statistically significant in all regions $(p<0.01)$.

showed that urothelial carcinoma had infiltrated the bilateral external and left obturator LNs (Fig. 2, parts F, G).

The LN intensity under in vivo probing was 3.01 for external iliac LNs, 4.35 for obturator LNs, and 7.30 for internal iliac LNs, and the intensity for the internal iliac nodes was significantly $(p<0.01)$ higher than the intensities for the other LNs (Fig. 3, part A). The intensities for the internal iliac and obturator nodes were significantly $(p<0.01)$ higher than the intensity for external iliac LNs (Fig. 3, part B). The $\mathrm{LN}$ intensity under in vivo probing was significantly higher in negative $L N$ metastasis than in positive $L N$ metastasis in internal iliac nodes $(p<0.05)$, but not in external iliac nodes and obturator nodes (Fig. 3, part C). The FN system appeared to be poor at identifying positive nodes compared to negative nodes, thus limiting its utility.

\section{Discussion}

The pathological stage of primary bladder cancer and the presence of LN metastasis are the most important determinants of survival in patients with bladder cancer undergoing radical cystectomy. ${ }^{10}$ In 1950, Leadbetter and Cooper ${ }^{6}$ 
described a technique for "regional gland dissection" that is still in use today. The limited LN dissection is generally described as an extirpation of lymphatic tissue in the obturator fossa between the obturator nerve and the external iliac vein. ${ }^{11}$ Another method of limited LN dissection, usually referred to as a conventional PLND ${ }^{12}$ or a standard LN dissection, ${ }^{13}$ includes the area stretching laterally as far as the genitofemoral nerve and posteriorly to the internal iliac vessels. It is apparent that the surgical approach for bladder cancer varies tremendously, and, despite a growing body of evidence to suggest that an aggressive surgical approach with an appropriate lymphadenectomy may benefit patients, this is not always performed. ${ }^{14}$ It is important to define and optimize the limits of PLNDs.

The conventional approach to determining LN involvement uses RIs, is technically difficult and uses potentially biohazardous agents. As such, we have developed an easier and more accurate technique in which ICG is injected into the bladder during radical cystectomy. ICG is a popular diagnostic reagent that is clinically approved for use in liver function tests. ${ }^{15}$ Several anaphylactoid reactions to ICG requiring resuscitation were reported in $0.05 \%$ of patients, ${ }^{16}$ while such reactions to patent blue dye were reported in $0.3 \%$ of patients. ${ }^{17}$ Our preliminary result was consistent with the previously reported results, and we have never seen adverse effects related to ICG injections. Our approach to ICG-fluorescence-guided lymphatic imaging is a simple, useful technique for PLND in bladder cancer patients.

The primary focus of this study was to identify the lymphatic channel that might be affected by the LN involvement of bladder cancer cells. The clinical superiority of the FN system to the Rl-assisted one is due to the ease of tracking the lymphatic vessels into the pelvic LNs under FN guidance. ${ }^{9}$ The results we obtained showed that the intensity of ICG fluorescence either in vivo or ex vivo was the strongest in the internal iliac region, second strongest in the obturator region, and weakest in the external iliac region. These results indicate that the major lymphatic channels involved in the spread of bladder cancer are those along the inferior vesical artery to the internal iliac artery. We think that obturator and external iliac nodes and the internal iliac region should be the target of PLND.

A secondary focus of this study was to determine whether the SLN concept is applicable to bladder cancer. In breast cancer, direct SLN biopsy under FN guidance using ICG is excellent, with a detection rate of $94 \% .{ }^{18}$ In our FN system, we were able to dissect and obtain $189 \mathrm{LNs}$, only 30 of which were positive for cancer cells (cases \#4, 5, 8, 9 and 11). In spite of the potential higher sensitivity of the FN system to LN involvement, these $30 \mathrm{LNs}$ were identified as negative for LN involvement during the operation and the false negative rate is $100 \%$. In cases \# 4, 5, 8 and 9, the primary lymphatic flow was obstructed by the infiltration of cancer cells and there was no strong fluorescence in the regional LNs of the bladder either in vivo or ex vivo. The pathological review of these 4 cases showed that alternative pathways develop when lymphatic pathways are completely obstructed by infiltrating cancer cells. In the case of a non-visualized LN rather than a brightly visualized one under the FN system, intra- and/or inter-communication of the lymphatic network around the bladder might accelerate induction of an alternative lymphatic pathway. Hence, intra-operative fluorescence is better suited to detect negative nodes than positive nodes and thus may not identify positive nodes. In the future, we will conduct intraoperative evaluations of lymphatic channels by using the FN system to find an optimal range of PLND in bladder cancer.

\section{Conclusions}

We have shown that using an FN system after injecting ICG into the bladder during radical cystectomy is a safe and easy approach to detecting the primary lymphatic channel draining from the bladder to optimize the range of PLND. The major lymphatic pathway involved in the spread of bladder cancer appears to be the one related to internal iliac LNs. Since the current results do not confirm that the SLN concept applies to bladder cancer, a validation study to determine whether SLN biopsy guided by FN is useful in bladder cancer is underway at our institution.

Competing interests: None declared.

This paper has been peer-reviewed.

\section{References}

1. Morton DL, Cochran AJ, Thompson JF, et al. Sentinel node biopsy for early-stage melanoma: accuracy and morbidity in MSLT-I, an international multicenter trial. Ann Surg 2005;242:302-11.

2. Posther KE, Wilke $L G$, Giuliano AE. Sentinel lymph node dissection and the current status of American trials on breast lymphatic mapping. Semin Oncol 2004;31:426-36. http://dx.doi.org/10.1053/i. seminoncol.2004.03.019

3. Cabanas RM. An approach for the treatment of penile carcinoma. Cancer 1977;39:456-66. http://dx.doi. org/10.1002/1097-0142(197702)39:2<456::AD-CNCR2820390214>3.0.C0;2-1

4. Wawroschek F, Vogt H, Weckermann D, et al. The sentinel lymph node concept in prostate cancer - first results of gamma probe-guided sentinel lymph node identification. Eur Urol 1999;36:595-600. http:// dx.doi.org/10.1159/000020054

5. Ito N, Fukuta M, Tokushima T, et al. Sentinel node navigation surgery using indocyanine green in patients with lung cancer. Surg Today 2004;34:581-5. http://dx.doi.org/10.1007/s00595-004-2780-y

6. Leadbetter WF, Cooper JF. Regional gland dissection for carcinoma of the bladder: a technique for one-stage cystectomy, gland dissection, and bilateral uretero-enterostomy. J Urol 1950;63:242-60.

7. Kitai $\mathrm{T}$, Inomoto $\mathrm{T}$, Miwa $M$, et al. Fluorescence navigation with indocyanine green for detecting sentinel lymph nodes in breast cancer. Breast Cancer 2005;12:21 1-5. http://dx.doi.org/10.2325/bbs.12.211

8. Mitsui Y, Shiina H, Arichi N, et al. Indocyanine green (ICG)-based fluorescence navigation system for discrimination of kidney cancer from normal parenchyma: application during partial nephrectomy. Int Urol Nephrol 2012; Epub ahead of printhttrp://dx.doi.org/10.1007/s1 1255-011-0120-x

9. Inoue $\mathrm{S}$, Shiina $\mathrm{H}$, Arichi $\mathrm{N}$, et al. Identification of lymphatic pathway involved in the spreading of prostate cancer by fluorescence navigation approach with intraoperatively injected indocyanine green. Can Urol Assoc J 2011;5:254-9. http://dx.doi.org/10.5489/cuaj.10159 
Inoue et al.

10. Stein JP, Lieskovsky G, Cote R, et al. Radical cystectomy in the treatment of invasive bladder cancer: long-term results in 1,054 patients. J Clin Oncol 2001;19:666-75.

11. Knap MM, Lundbeck F, Overgaard J. The role of pelvic lymph node dissection as a predictive and prognostic factor in bladder cancer. Eur J Cancer 2003;39:604-13. http://dx.doi.org/10.1016/ S0959-8049(02)00768-2

12. Frank I, Cheville JC, Blute ML, et al. Transitional cell carcinoma of the urinary bladder with regional lymph node involvement treated by cystectomy: clinicopathologic features associated with outcome. Cancer 2003;97:2425-31. htrp://dx.doi.org/10.1002/cncr.11370

13. Bochner BH, Cho D, Herr HW, et al. Prospectively packaged lymph node dissections with radical cystectomy: evaluation of node count variability and node mapping. J Urol 2004;172:1286-90. http://dx.doi. org/10.1097/01.ju.0000137817.56888.dl

14. Stein JP. Lymphadenectomy in bladder cancer: how high is "high enough"? Urol Oncol 2006;24:349-55. http://dx.doi.org/10.1016/i.urolonc.2005.07.013
15. Kusano M, Tajima Y, Yamazaki K, et al. Sentinel node mapping guided by indocyanine green fluorescence imaging: a new method for sentinel node navigation surgery in gastrointestinal cancer. Dig Surg 2008;25:103-8. http://dx.doi.org/10.1159/000121905

16. Olsen TW, Lim Jl, Capone A Jr, et al. Anaphylactic shock following indocyanine green angiography. Arch Ophthalmol 1996;114:97. http://dx.doi.org/10.1001/archopht.1996.01100130093018

17. Beenen E, de Roy van Zuidewiin DB. Patients blue on patent blue: an adverse reaction during four sentinel node procedures. Surg Oncol 2005;14:151-4. http://dx.doi.org/10.1016/i.suronc.2005.12.001

18. Motomura K, Inaii H, Komoike Y, et al. Combination technique is superior to dye alone in identification of the sentinel node in breast cancer patients. J Surg Oncol 2001;76:95-9.

Correspondence: Dr. Shogo Inoue, Department of Urology, Graduate School of Biomedical Sciences, Hiroshima University, 1-2-3 Kasumi, Minami-ku, Hiroshima 734-8551; fax: -81-82-257-5244; inosyogo@hiroshima-u.ac.jp 